patient and physician satisfaction and greater overall work impairment compared with non-flaring patients. There is a need for more effective treatments in this patient population to reduce patient and healthcare burden.

Study funded by Johnson and Johnson.

Disclosure of Interests: Zahi Touma Consultant of: Consultant for Janssen, Ben Hoskin Consultant of: Consultant for Janssen, Christian Atkinson Consultant of: Consultant for Janssen, David Bell Consultant of: Janssen, Olivia Massey Consultant of: Janssen, Jennifer H. Lofland Employee of: Janssen, Pamela Berry Employee of: Janssen, Chetan Karyekar Shareholder of: Johnson \& Johnson, Consultant of: Janssen, Employee of: Janssen Global Services, LLC. Previously, Novartis, Bristol-Myers Squibb, and Abbott Labs., Karen Costenbader Grant/ research support from: Merck, Consultant of: Astra-Zeneca

DOI: 10.1136/annrheumdis-2020-eular.6010

\section{SAT0214 ULTRASONOGRAPHIC CHANGES OF SALIVARY GLANDS IN PRIMARY SJOGREN'S SYNDROME: A LONGITUDINAL PROSPECTIVE STUDY}

H. R. Kim ${ }^{1}$, K. A. Lee ${ }^{2}$, S. H. Lee ${ }^{1}$, S. H. Kim ${ }^{1}{ }^{1}$ Konkuk University School of Medicine, Seoul, Korea, Rep. of (South Korea); ${ }^{2}$ Soonchunhyang University Seoul Hospital, Seoul, Korea, Rep. of (South Korea)

Background: In the diagnosis of primary Sjogren' syndrome (SS), salivary gland ultrasound is useful tool. Until now, there is no data for ultasonographic changes of major salivary glands over time.

Objectives: This study aimed to evaluate the changes in abnormalities of salivary gland ultrasound (SGUS) over time in patients with pSS.

Methods: Patients with pSS $(n=70)$ and idiopathic sicca syndrome $(n=18)$ underwent SGUS twice at baseline and 2 years later. The semi-quantitative SGUS score (0-48) was used, which comprises five parameters: parenchymal echogenicity, homogeneity, hypoechoic areas, hyperechogenic reflections, and clearness of posterior borders. The intraglandular power Doppler signal (PDS) was also assessed. The changes of these SGUS variables were compared in patients with pSS and idiopathic sicca syndrome.

Results: The median (interquartile range) total SGUS scores at baseline was 27 (14) in patients with and 4 (3) in those with idiopathic sicca syndrome $(p<0.001)$. In the pSS group, the total SGUS scores and the SGUS scores for bilateral parotid glands were significantly increased during median 23.4 month follow-up ( $p=0.013$ and $p=0.011$, respectively). Homogeneity and hypoechoic areas were the domain to show statistically significant progression of SGUS scores. None of the SGUS scores changed significantly in the patients with idiopathic sicca syndrome. In patients with pSS, baseline and follow-up PDS sum scores of four salivary glands were significant higher in worsening SGUS group $(n=13)$ than no change/improvement SGUS group $(n=55 / 2)$.

Conclusion: The structural abnormalities in major salivary glands assessed using SGUS scores progressed significantly in patients with pSS. In pSS group, $18.6 \%$ patients had worsening SGUS scores during 2 years. Intra-glandular hypervascularity was associated with worsening of salivary gland abnormalities. References:

[1] Delli K, Dijkstra PU, Stel AJ, Bootsma H, Vissink A, Spijkervet FK. Diagnostic properties of ultrasound of major salivary glands in Sjogren's syndrome: a meta-analysis. Oral diseases. 2015;21(6):792-800.

[2] Jousse-Joulin S, Devauchelle-Pensec V, Cornec D, Marhadour T, Bressollette L, Gestin S, et al. Brief Report: Ultrasonographic Assessment of Salivary Gland Response to Rituximab in Primary Sjogren's Syndrome. Arthritis \& rheumatology (Hoboken, NJ). 2015;67(6):1623-8.

[3] Gazeau P, Cornec D, Jousse-Joulin S, Guellec D, Saraux A, Devauchelle-Pensec V. Time-course of ultrasound abnormalities of major salivary glands in suspected Sjogren's syndrome. Joint, bone, spine: revue du rhumatisme. 2018;85(2):227-32.

[4] Lee KA, Lee SH, Kim HR. Diagnostic and predictive evaluation using salivary gland ultrasonography in primary Sjogren's syndrome. Clinical and experimental rheumatology. 2018;36 Suppl 112(3):165-72.

Acknowledgments: : This work was funded by the Konkuk University Medical Center Research Grant 2019.

Disclosure of Interests: None declared

DOI: 10.1136/annrheumdis-2020-eular.337

\section{SAT0215 \\ HISTORY OF TONSILLECTOMY IS ASSOCIATED WITH GLANDULAR INFLAMMATION IN SJÖGREN'S SYNDROME}

F. Kollert $^{1,2}$, V. Pucino ${ }^{2,3}$, S. Rauz ${ }^{2,4}$, A. Richard ${ }^{5}$, J. Higham ${ }^{5}$, A. PovedoGallego $^{2,5}$, R. M. Brown ${ }^{6}$, T. Bates ${ }^{6}$, S. J. Bowman ${ }^{2,3}$, F. Barone ${ }^{2,3}$, B. Fisher ${ }^{2,3}$. ${ }^{1}$ Department of Rheumatology, Immunology and Allergology, Inselspital, University Hospital Bern, Bern, Switzerland; ' Institute of Inflammation and Aeging, College of Medical and Dental Sciences, University of Birmingham, Birmingham, United Kingdom; ${ }^{3}$ National Institute for Health Research (NIHR),
Birmingham Biomedical Research Centre and Department of Rheumatology, University Hospitals Birmingham NHS Foundation Trust, Birmingham, United Kingdom; ${ }^{4}$ Academic Unit of Opthalmology, Birmingham and Midland Eye Centre, Birmingham, United Kingdom; ${ }^{5}$ Department of Oral Medicine, Birmingham Dental Hospital, Birmingham, United Kingdom; ${ }^{6}$ Department of Histopathology, University Hospitals Birmingham NHS Foundation Trust, Birmingham, United Kingdom

Background: The palatine tonsils are secondary lymphoid-organs that serve as the first line of defense against pathogens. Whether history of tonsillectomy (TE) is associated with the phenotype of Sjögren`s syndrome (SjS) has not been investigated to date.

Objectives: To test whether TE is linked to SjS phenotype and disease activity scores.

Methods: A total of 183 patients from the Optimising Assessment in Sjögren`s Syndrome (OASIS) cohort with SjS or non-SjS sicca syndrome were analysed. Patients with SjS fulfilled 2016 ACR/EULAR classification for primary SjS; sicca patients had objective and/or subjective dryness, but were anti-Ro/SSA negative and had no physician diagnosis of SjS. One SjS patient who had TE around the time of symptom onset was excluded.

Results: Of the total cohort, 116 were diagnosed with SjS (86.2\% SSA/Ro positive) and 67 with non-SjS sicca syndrome. Overall, 29\% (53/183) had TE; $24.1 \%$ of the SjS patients $(28 / 116)$ and $37.3 \%$ of the sicca patients $(25 / 67)$. The prevalence of TE was higher in sicca than in SjS $(p=0.043)$. The median age at TE was 8 (range 3-50) years and did not differ between SjS and sicca patients $(p=0.629)$ Neither age at first symptoms $(p=0.093)$ nor disease duration $(p=0.623)$ were associated with TE in patients with SjS. SjS patients with TE showed a higher average histological focus score $(2.1(1.2-2.8)$ vs. $1.3(0.0-4.3) ; p=0.049)$, and were more likely to have activity in the glandular (53.6 vs. $20.5 \% ; p=0.001$ ) and constitutional ( 39.3 vs. $14.9 \%, p=0.014)$ domains of the ESSDAI, and lower levels of $\operatorname{lgG}(12.2(7.8-35.6)$ vs. $15.6(5.7-56.4) \mathrm{g} / \mathrm{l} ; \mathrm{p}=0.012)$ and $\lg \mathrm{A}(2.3(0.9-6.6)$ vs. $2.9(0.7-9.4) \mathrm{g} / \mathrm{l} ; \mathrm{p}=0.032)$. Whereas there was no difference in EQ5D utility values $(p=0.718)$, VAS global health was significantly lower in the patients with SjS who had TE (58 (10-78) vs. 70 (10-97); $p=0.021)$. There was no association between the status of TE and autoantibodies (SSA, SSB, RF), lachrymal and salivary glands function (Schirmer's test, unstimulated saliva flow), complement (C3, C4), serum levels of free light chains, $\beta 2$-microglobulin, ESSPRI, or tota ESSDAI (all p-values $>0.1)$. Of 181 patients, $12.7 \%(23 / 283)$ had appendectomy $(A E) ; 10.5 \%(12 / 114)$ of the SjS patients and $16.4 \%(11 / 67)$ of the sicca patients $(p=0.258)$. With the exception of lower unstimulated salivary flow $(0.086(0.01$ $0.43)$ vs. $0.11(0.0-1.3) \mathrm{ml} / \mathrm{min} ; \mathrm{p}=0.026)$ in $\mathrm{SjS}$ patients with $A E$, there were no differences in disease phenotype between $\mathrm{SjS}$ patients with and without $A E$ (all p-values $>0.1$ ).

Conclusion: History of TE in SjS is associated with higher average focus scores and with glandular swelling. It could be speculated that the absence of palatine tonsils is compensated by enhanced lymphocytic infiltrates in the salivary glands. Further research is required to determine if TE is a risk factor for both SjS and non-SjS sicca and to determine the role of the tonsils in the generation of hypergammaglobulinaemia in SjS.

Disclosure of Interests: Florian Kollert Employee of: Novartis, Valentina Pucino: None declared, Saaeha Rauz: None declared, Andrea Richard: None declared, Jon Higham: None declared, Ana Povedo-Gallego: None declared, Rachel M. Brown: None declared, Timothy Bates: None declared, Simon J. Bowman Consultant of: Astrazeneca, Biogen, BMS, Celgene, Medimmune, MTPharma, Novartis, Ono, UCB, xtlbio, Glapagos, Speakers bureau: Novartis, Francesca Barone: None declared, Benjamin Fisher: None declared

DOI: 10.1136/annrheumdis-2020-eular.2112

\section{SAT0216 DISEASE SEVERITY, COMORBID CONDITIONS, TREATMENT PATTERNS, AND FLARES IN ADULTS WITH SYSTEMIC LUPUS ERYTHEMATOSUS IN THE UNITED KINGDOM: A REAL-WORLD OBSERVATIONAL RETROSPECTIVE COHORT ANALYSIS}

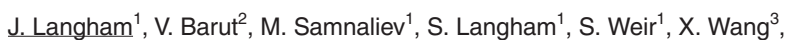
B. Desta4, E. R. Hammond4. ${ }^{1}$ Maverex Limited, Manchester, United Kingdom; ${ }^{2}$ BioPharmaceuticals Medical, AstraZeneca, Cambridge, United Kingdom; ${ }^{3}$ BioPharmaceuticals Medical, AstraZeneca, Gaithersburg, United States of America; ${ }^{3}$ BioPharmaceuticals Medical, AstraZeneca, Gaithersburg, United States of America

Background: There is limited real-world evidence describing the presentation and treatment patterns of systemic lupus erythematosus (SLE) in the United Kingdom (UK).

Objectives: To characterize disease severity, comorbid conditions, treatment patterns, and flares in a longitudinal cohort of adults with SLE in the UK. 